\title{
Wide-area Detection of Voltage Instability from Synchronized Phasor Measurements. Part II: Simulation results
}

\author{
Mevludin Glavic, Senior Member, IEEE
}

\begin{abstract}
This two-part paper deals with the early detection of an impending voltage instability from the system states provided by synchronized phasor measurements. Recognizing that voltage instability detection requires assessing a multidimensional system, the method fits a set of algebraic equations to the sampled states, and performs an efficient sensitivity in order to identify when a combination of load powers has passed through a maximum. This second part of the paper presents simulation results obtained from detailed time-domain simulation of the Nordic32 test system, without and with measurement noise, respectively. Several practical improvements are described such as anticipation of overexcitation limiter activation, and use of a moving average filter. Robustness to load behaviour, non updated topology and unobservability is also shown. Finally a comparison with Thévenin impedance matching criterion is provided.
\end{abstract}

Index Terms-Long-term voltage stability, instability detection, wide-area monitoring, phasor measurement units, sensitivity analysis

\section{INTRODUCTION}

$\mathbf{T}$ His paper is the continuation of [1]. Section II reports on the method validation using bus voltage phasors provided by time-domain simulation and hence affected by short-term, electromechanical transients, but no measurement noise. The effect and the filtering of the latter are considered in Section III. Next, the improved detection capability obtained when anticipating overexcitation limiter (OEL) activation is demonstrated in Section IV while Section V reports on several robustness tests. Finally, a comparison with Thévenin impedance matching criterion is provided in Section VI. Section VII is devoted to concluding remarks and directions for future work.

\section{RESUlTS IN THE ABSENCE OF MEASUREMENT NOISE}

\section{A. Test system}

We report on results obtained with a slightly modified version of the so-called Nordic32 test system, detailed in [2]. The one-line diagram of this 52-bus, 20-machine system is shown in Fig. 1.

The model includes a detailed ( 3 or 4 rotor winding) representation of each synchronous machine, and a generic model of speed governor (for generators in the North and Equiv areas;

M. Glavic (glavic@montefiore.ulg.ac.be) is visiting professor, supported by an FNRS (Fund for Scientific Research) grant, at the Dept. of Electrical Engineering and Computer Science of the University of Liège, Sart Tilman B37, B-4000 Liège, Belgium.

T. Van Cutsem (t.vancutsem@ulg.ac.be) is a research director of FNRS and adjunct professor at the same department.

\author{
Thierry Van Cutsem, Fellow, IEEE
}

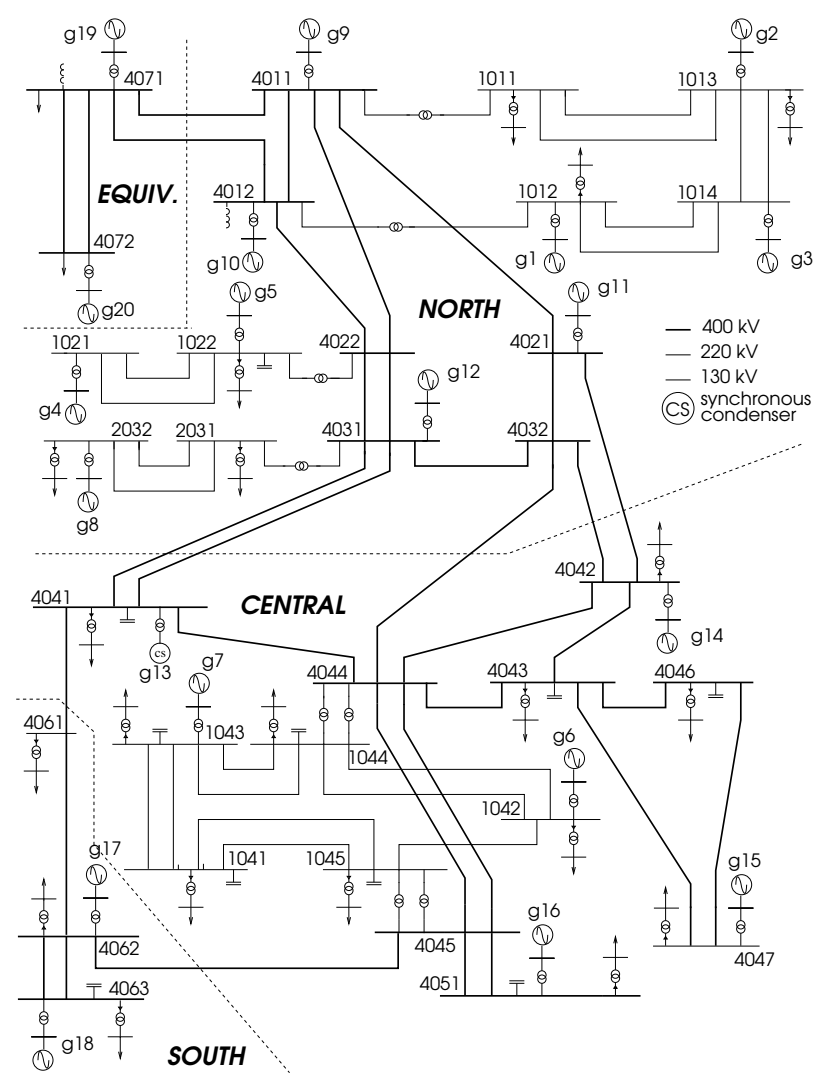

Fig. 1. One-line diagram of Nordic32 test system

the other ones do not participate in frequency control), hydro or steam turbine, AVR and OEL. The latter is of the nontakeover type and obeys an inverse-time characteristic [3].

The loads behave as constant current for the active power and constant impedance for the reactive power. Each one is fed through a transformer with automatic LTC. There is a delay of 30 seconds on the first tap change while the delay on subsequent tap changes varies from 8 to $12 \mathrm{~s}$ from one LTC to another.

\section{B. PMU output simulation}

Time-domain simulations of the model have been performed with the Simulink-based variable-step integration software outlined in [4]. This provides the vectors $\mathbf{v}_{x}$ and $\mathbf{v}_{y}$ at successive time instants, referred to axes rotating at nominal angular frequency. These values have been interpolated and sampled every $0.1 \mathrm{~s}$. Furthermore, at each sampling time, the 


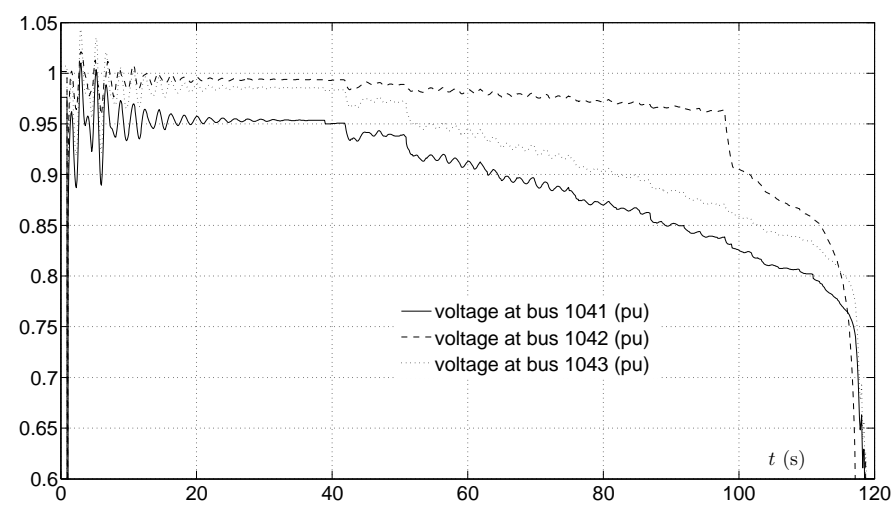

Fig. 2. Case A: voltages at buses 1041, 1042 and 1043

phase angle of the voltage at the reference bus 4072 has been subtracted from all voltage phase angles. This is reflected in the equation: $v_{y}=0$ at bus 4072, which is embedded in the calculation of the Jacobian $\varphi_{\mathrm{z}}$.

Communications delays and state estimation computing times have not been considered since they are not critical in this application.

\section{Simulation results}

For the sake of clarity, we first illustrate the performance of the method when the (simulated) measurements are affected by significant transients but no additional random noise.

1) Case A: unstable scenario: We first consider a stressed operating condition with heavy power transfers from North and Equiv. to Central and South areas (see Fig. 1). Most of generators in Central area operate close to their OEL limits, i.e. with insufficient reactive power reserves. The contingency considered is a three-phase short circuit at $t=0.95 \mathrm{~s}$ on line 4032-4044, near bus 4044, cleared by opening the faulted line after $0.1 \mathrm{~s}$.

This disturbance makes the system long-term voltage unstable. Figure 2 shows the evolution of voltage magnitudes at the three most affected buses, located in Central area. The system evolves over some 120 seconds under the effect of LTCs trying unsuccessfully to restore the distribution voltages (and hence the load powers), as well as OELs acting successively over: $\mathrm{g} 5$ at $t=30.8 \mathrm{~s}, \mathrm{~g} 14$ at $43.1 \mathrm{~s}, \mathrm{~g} 12$ at $51.7 \mathrm{~s}, \mathrm{~g} 7$ at $52.1 \mathrm{~s}$, $\mathrm{g} 15$ at $52.3 \mathrm{~s}, \mathrm{~g} 16$ at $t=57.9 \mathrm{~s}$ and eventually g6 at $99.1 \mathrm{~s}$, thereby further reducing the power that can be transmitted to loads. The long-term voltage instability results in a loss of synchronism of field current limited generators, soon after g6 gets limited.

The evolution of a sample of $S_{Q_{q} Q_{j}}$ sensitivities is shown in Fig. 3. A typical value before disturbance is 2, at most 4 . The change in the sign takes place at $t=51.5$ s, i.e. 50.5 seconds after fault clearing and 67.3 seconds before collapse. Note that all transmission voltages are still above $0.92 \mathrm{pu}$ at that time. Thus, the developing instability is detected quite early. Further anticipation is possible, as shown in Section IV.

The sensitivities oscillate under the effect of electromechanical transients. The peaks can be related to important voltage dips. Although not critical, these oscillations could be filtered

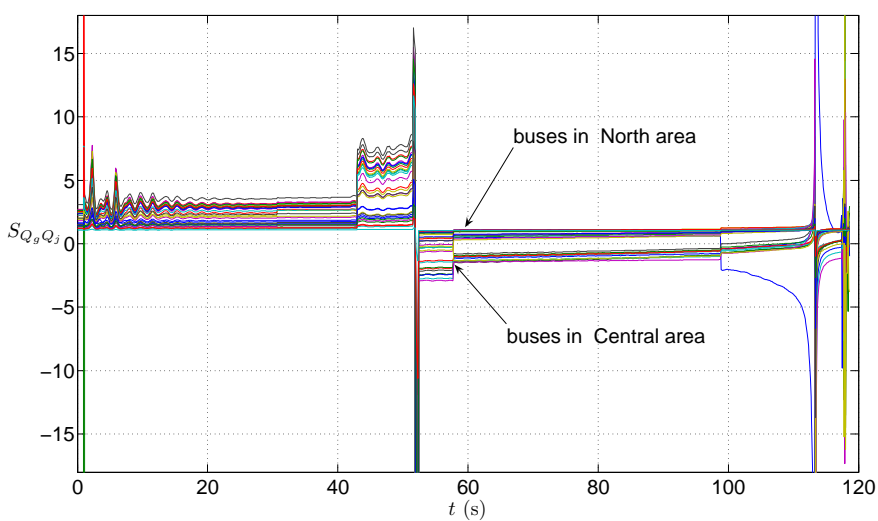

Fig. 3. Case A: sensitivities $S_{Q_{g} Q_{j}}$ at various buses

out as discussed in Section III. One sensitivity changes sign near $t=1 \mathrm{~s}$, under the effect of the fault, but this is easily identified as a short transient. Eventually, many sensitivities change sign after $t=110 \mathrm{~s}$ when some machines start getting out of step. One can see the "signature" of another eigenvalue passing through zero. Anyway, this takes place when the system is clearly in emergency as seen from the unacceptably low voltages.

All sensitivities but a few relative to the North area, change sign together. Some eight seconds after, some of them relative to buses in North change back to positive, but without passing through large values. On the contrary, those relative to Central area remain negative. This behaviour, due to eigenvalue movement, is in perfect agreement with the results in [5], obtained under the Quasi Steady-State (QSS) time simulation. From a practical viewpoint, it is important to note that the negative sensitivities point to the collapsing Central area.

2) Case B: marginally unstable situation: The system operates in less stressed conditions after load has been decreased by $210 \mathrm{MW}$ and $70 \mathrm{Mvar}$ in the Central area. This amount has been chosen to make the system marginally unstable. The disturbance is the tripping at $t=1 \mathrm{~s}$ of the same line, without fault.

The voltage evolution is shown with heavy line in Fig. 4. The marginal character of the case is seen from the much longer time the system takes to collapse. OELs act over generators $\mathrm{g} 14, \mathrm{~g} 7, \mathrm{~g} 12, \mathrm{~g} 15, \mathrm{~g} 16$ and $\mathrm{g} 6$ at $t=$ $89.2,132.8,369.6,440.2,530.2$, and $611.9 \mathrm{~s}$, respectively.

The corresponding sensitivity evolution is shown in Fig. 5. The change in sign happens $439 \mathrm{~s}$ after the line outage and $190 \mathrm{~s}$ before collapse, thus giving an early warning of the slowly developing instability.

3) Case C: marginally stable situation: A marginally stable case has been produced by decreasing the load in Central area by $250 \mathrm{MW}$ and $80 \mathrm{Mvar}$ with respect to Case A. The disturbance is the tripping at $t=1 \mathrm{~s}$ of the same line.

The voltage evolution is shown with dotted lines in Fig. 4. The system takes some $320 \mathrm{~s}$ to settle at a long-term equilibrium. OELs are activated on generators g7 and g14 only, at $t=106.2$ and $107.2 \mathrm{~s}$, respectively.

The sensitivity evolution is given in Fig. 6. Although the sensitivities rise to values that denote a weak operating 


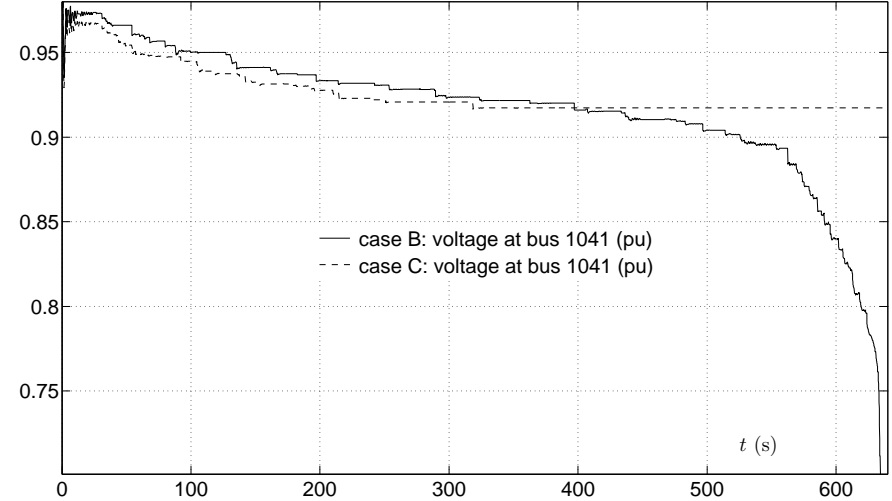

Fig. 4. Cases B and C: Voltage at bus 1041

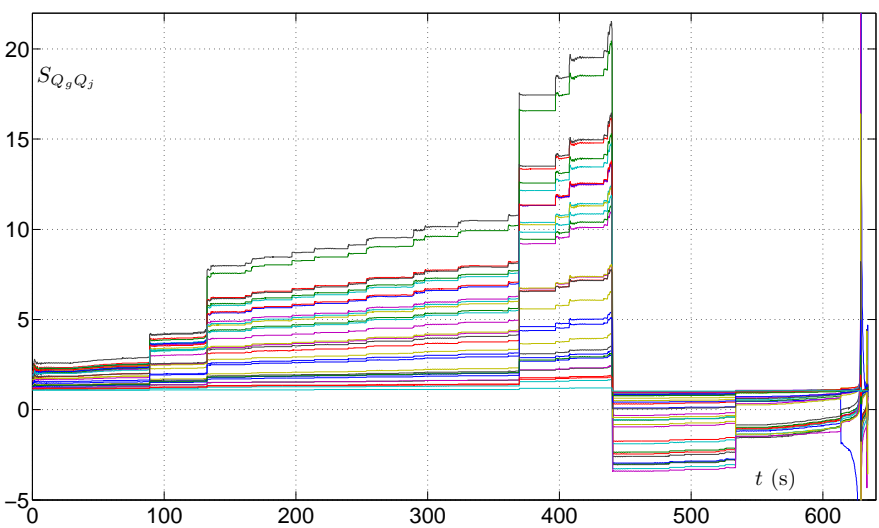

Fig. 5. Case B: sensitivities $S_{Q_{g} Q_{j}}$ at various buses

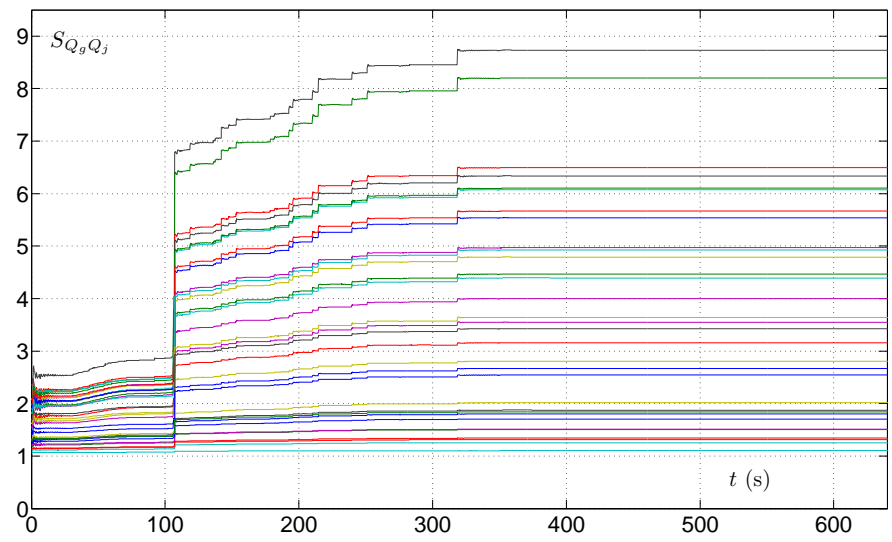

Fig. 6. Case C: sensitivities $S_{Q_{g} Q_{j}}$ at various buses

condition, no one changes sign; hence, no false alarm is issued in this marginally stable case.

The proposed method thus shows promise in terms of dependability and security.

\section{RESULTS IN THE PRESENCE OF MEASUREMENT NOISE}

Despite high PMU accuracy, in real applications, there will remain measurement errors due to relatively unchanged current and potential transformers (although the future may bring improvements such as optical/digital devices) [6], [7].

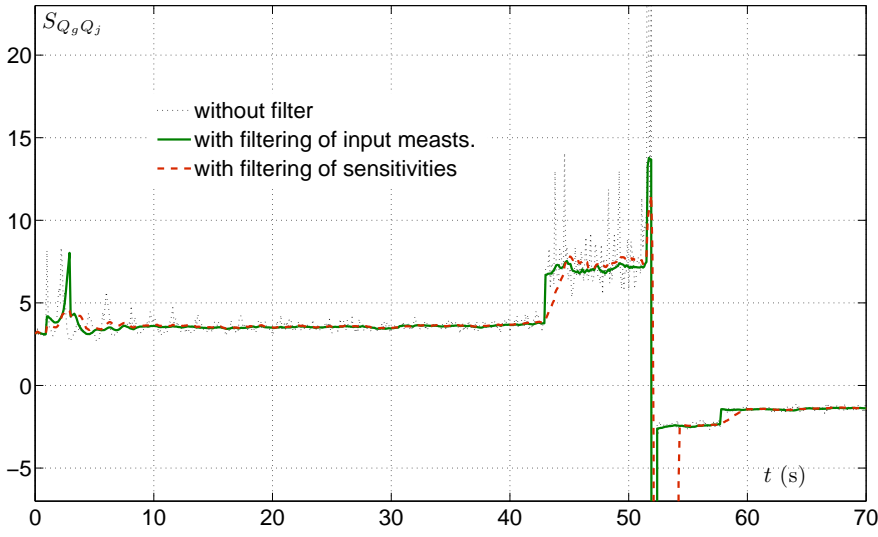

Fig. 7. Sensitivity $S_{Q_{g} Q_{j}}$ at bus 1041 in case A; noise $N(0,0.01)$ added to all voltage components

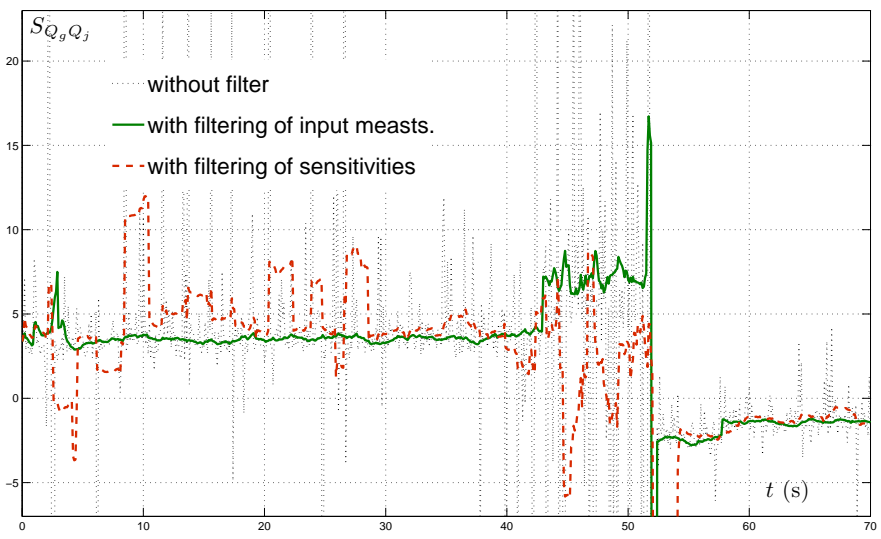

Fig. 8. Sensitivity $S_{Q_{g} Q_{j}}$ at bus 1041 in case A; noise $N(0,0.04)$ added to all voltage components

Preprocessing data by a (linear) state estimator will somewhat reduce the noise effect but filtering is needed in any case to deal with fast transients, not taken into account in the algebraic model. Filtering is even more needed if voltage phasors are provided by PMUs directly.

Measurement inaccuracy was simulated by simply adding a random value to each component of the $\mathbf{v}_{x}$ and $\mathbf{v}_{y}$ vectors, at each sample time. This random variable has a Gaussian distribution $N(0, \sigma)$. For simplicity, no correlation was assumed between both components of the same voltage.

\section{A. Results without filter}

We first consider a "reasonably low" measurement noise, characterized by $\sigma=0.01$ pu. The dotted line in Fig. 7, relative to Case A, shows the evolution of the sensitivity at bus 1041 (exhibiting the largest magnitude). Expectedly, larger fluctuations are observed when approaching the maximum load power point, but the latter is still easily identified.

Next, we consider a "high" measurement noise, characterized by $\sigma=0.04 \mathrm{pu}$. The sensitivity at bus 1041 varies as shown with dotted line in Fig. 8. With this important level of noise, the sensitivities undergo many spurious changes in sign, and interpretation is impossible. 


\section{B. Choice of a filter}

Various digital filters were tested. Nice performances were obtained with a simple Moving Average (MA) filter. As the name implies, this operates by averaging a number of past inputs. Thus, at each discrete time step $k$, it produces an output defined by:

$$
\bar{z}(k)=\frac{z(k)+z(k-1)+\ldots+z(k-N+1)}{N}
$$

where $N$ is the size of the moving window, related to cut-off frequency. The MA filter is known for good performances in preserving sharp step changes of the inputs. Our tests were performed with $N=20$, except for $t \in\{0,0.1,0.2, \ldots, 1.9\}$ where the available samples were used.

\section{Results with filter}

The curves shown with dashed lines in Figs. 7 and 8 have been obtained by applying the MA filter to the sensitivities themselves. The curves are smoother but the one in Fig. 8 does not show a clear trend.

Finally, the curves shown with heavy lines in Figs. 7 and 8 have been obtained by applying the MA filter to the input voltage phasors. At both noise levels, the curves are similar to the one obtained without noise (see Fig. 3) and the change in sign is clear.

It is thus recommended to apply the MA filter to input voltage phasors, especially if raw PMU data are used. This is appropriate even if a state estimator is used to obtain the voltages, since the latter may still be impacted by measurement inaccuracies.

The disadvantage of the MA filter is to introduce a delay. In the above examples, it is not larger than $1.9 \mathrm{~s}$, which has very little impact in the application of concern. Nevertheless, some optimization of the window size could be considered.

All results shown in the sequel were obtained with the $N(0,0.01)$ random noise added to all voltage components and the MA filter applied to the so obtained input measurements.

\section{INCREASING ANTICIPATION CAPABILITY}

\section{A. Anticipating OEL activation}

As explained in the companion paper [1], an estimate of $E_{q}$, the e.m.f. proportional to field current, is used to identify whether a synchronous generator operates under control of its AVR or has its field current limited by its OEL. Under AVR control, an equation such as:

$$
k E_{q}^{s}-G\left(V^{o}-\sqrt{v_{x}^{2}+v_{y}^{2}}\right)=0
$$

is used, while under OEL control, it is replaced by an equation of the type:

$$
k E_{q}^{s}-E_{q}^{l i m}=0
$$

Furthermore, it is of interest to anticipate the effect of an approaching OEL activation [8]. To this purpose, when $E_{q}>$ $E_{q}^{l i m}+\epsilon$, the OEL equation (3) is anticipatively substituted to the AVR equation (2) when evaluating the Jacobian $\varphi_{z}$. This remains in effect as long as the OEL is acting, which is identified by $E_{q}^{l i m}-\epsilon \leq E_{q} \leq E_{q}^{l i m}+\epsilon$.

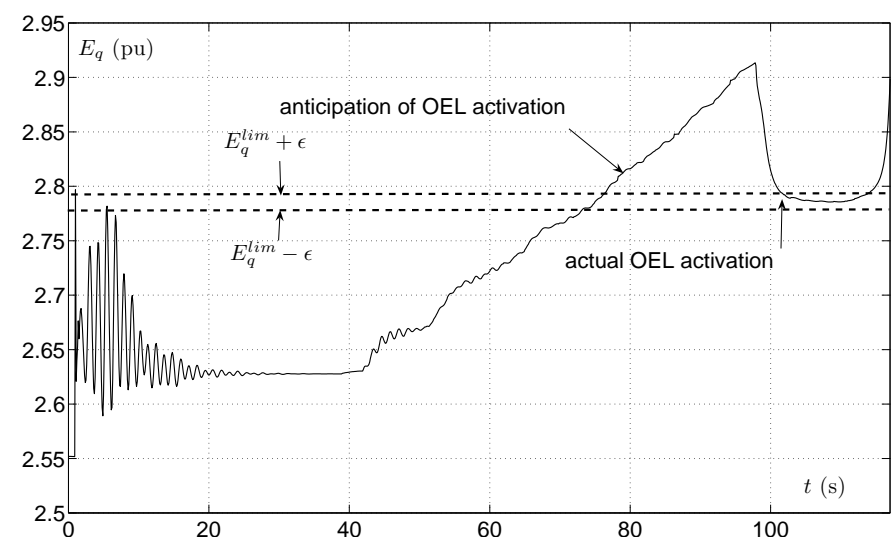

Fig. 9. Calculated value of e.m.f. $E_{q}$ of generator g6 (in pu)

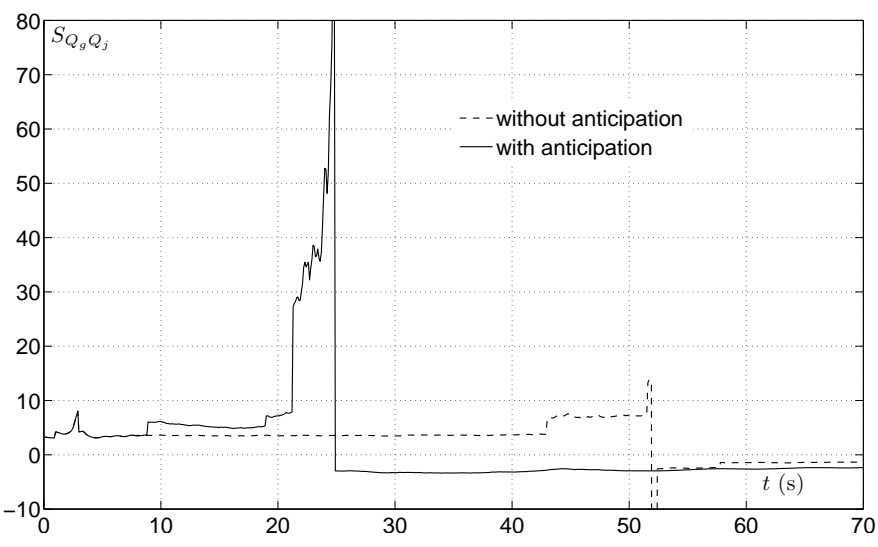

Fig. 10. Case A: sensitivity $S_{Q_{g} Q_{j}}$ at bus 1041 with and without anticipation of OEL activation

In practice, since $E_{q}$ may undergo large but short-lasting changes under the effect of electromechanical transients, the inequality $E_{q}>E_{q}^{l i m}+\epsilon$ has to hold true for some period of time $\tau$ before the equation switching takes place.

Further anticipation could be obtained by determining the operating point that will result from the field current enforcement, and computing the Jacobian at that point. By not considering this option here, we accepted some inconsistency between the system state and the equations used to build the Jacobian. This was found to somewhat amplify sensitivity variations but with no practical impact.

\section{B. Results}

Figure 9 shows the evolution of the e.m.f. $E_{q}$ of generator g6, in case A, computed as detailed in [1]. The figure also shows the time at which future OEL activation is anticipated, and the OEL equation (3) already substituted to the AVR equation (2) when evaluating the Jacobian. $\tau$ has been set to 3 seconds, to avoid reacting to electromechanical transients with some security margin. $\epsilon$ has been set to $0.01 \mathrm{pu}$, the value of $E_{q}^{l i m}$ being 2.79 pu on the same voltage base.

A comparison of sensitivity evolution with and without this technique is provided in Figs. 10, 11 and 12, showing the sensitivity at bus 1041 in Cases A, B, and C, respectively. In Case A, the instability is detected 26.5 seconds earlier (i.e. 


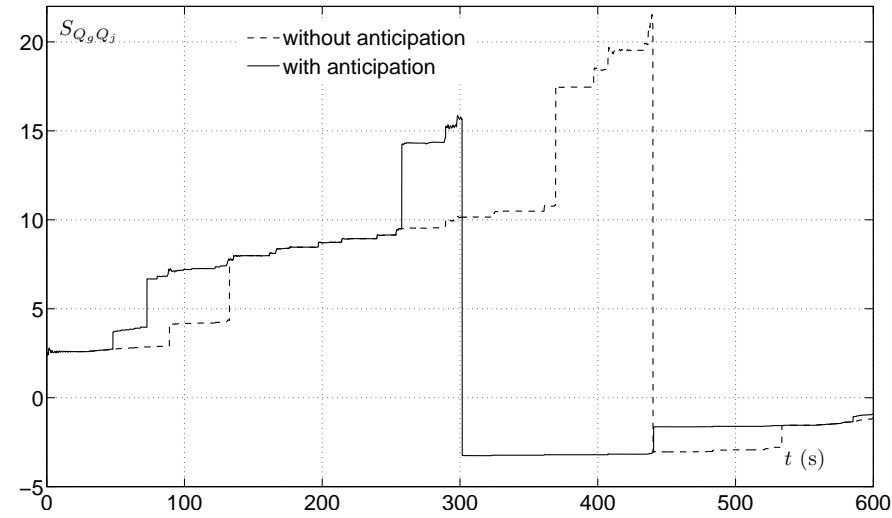

Fig. 11. Case B: sensitivity $S_{Q_{g} Q_{j}}$ at bus 1041 with and without anticipation of OEL activation

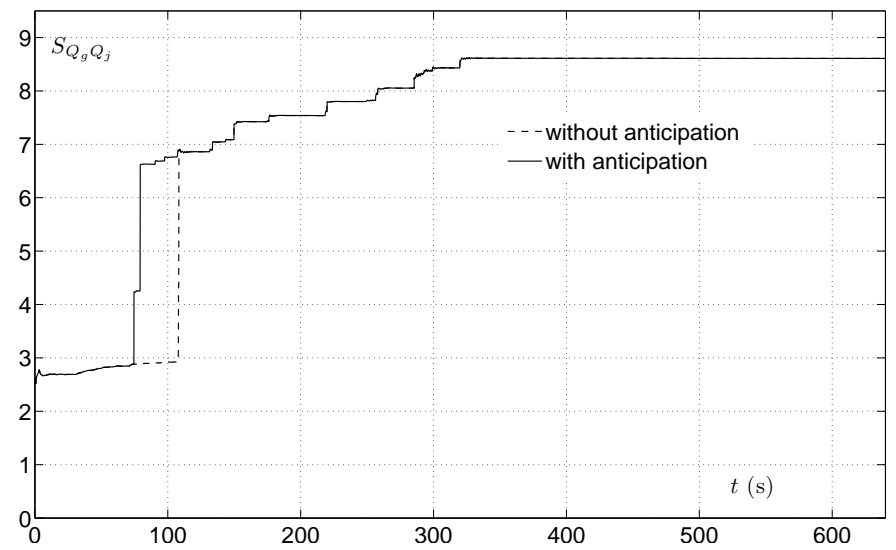

Fig. 12. Case C: sensitivity $S_{Q_{g} Q_{j}}$ at bus 1041 with and without anticipation of OEL activation

$104.5 \mathrm{~s}$ before collapse). In the marginally unstable Case B, detection takes place 140.4 seconds earlier (i.e. $331 \mathrm{~s}$ before collapse). In the marginally stable Case C, OEL activations on generators $\mathrm{g} 7$ and $\mathrm{g} 14$ are anticipated at $\mathrm{t}=74.7$ and 78.6 seconds (activations take place at 107.1 and $108.2 \mathrm{~s}$ ). This makes the sensitivity increase earlier but it reaches the same final value, and no false alarm is issued. There is little risk of wrongly detecting instability in so far as the technique is applied only to generators which indeed are going to be limited.

\section{RoBUStNess TO MODELLING ASSUMPTIONS}

\section{A. Performance in the presence of motor loads}

No load model is required in the proposed sensitivity calculation. Indeed, the objective is to identify a combination of load powers passing through a maximum. As shown in [1], this leads to treating loads as constant power when performing sensitivity analysis at one point in time (of course, those powers change from one snapshot to another). The load dynamics (motor speed changes, load tap changers, thermostatic load recovery, etc.) are accounted for through the change in voltage phasors which they contribute to.

In the examples so far, an exponential load model has been considered at the distribution buses controlled by LTCs. It was

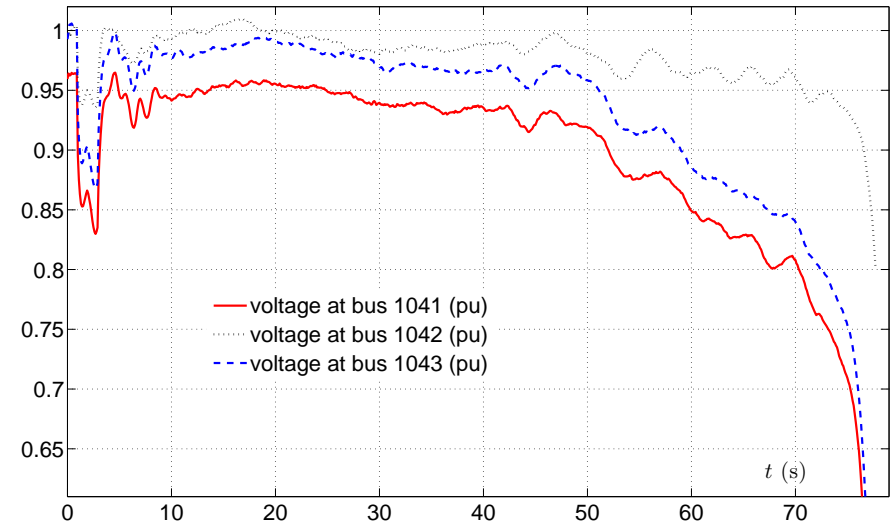

Fig. 13. voltage evolutions; Case A with $50 \%$ motor load

of interest to test the method in the presence of induction motor loads. It is well-known that the stalling of motors under low voltage conditions may precipitate system collapse. Motors may stall under the effect of short-circuits cleared too slowly [3], [9]; the resulting short-term voltage instability is, however, not addressed by the proposed method. In the case of longterm voltage instability, motors may stall when voltages are no longer supported owing to OEL activation [3], [9].

The example provided hereafter refers to a case where half of the initial power at the critical buses 1041, 1042, 1043,1044 , and 1045 is consumed by induction motors. One equivalent motor representing a population of small industrial motors has been considered at each bus, with the parameters provided in [9].

Figure 13 shows the evolution of voltages at three of the five buses under the effect of the same disturbance as in Case A. Note that the voltage dip caused by the initial short circuit is smoothed by the MA filter. The system collapses significantly faster. Voltages fall sharply, especially at bus 1042. Hence, much less time is left to a system protection scheme that would rely on the sole voltages, and anticipation becomes essential.

The sensitivity evolution, with and without OEL anticipation, is presented in Fig. 14. The change in sign is clearly identified. Furthermore, anticipating OEL activation allows to detect the impending stability some $27 \mathrm{~s}$ earlier, at a time where voltages still have quite normal values.

Figure 15 shows the effect of varying the proportion $p$ of motor load (at the above mentioned five buses uniformly). The diagram stops at $p=0.70$, since for higher proportions of motor load, short-term voltage instability takes place. The collapse time decreases when $p$ increases, because the faster load power recovery makes OELs react faster. Without OEL anticipation, the instability detection time is almost constant, and relatively little time is left at high values of $p$. On the other hand, with OEL anticipation, collapse is diagnosed at least $34 \mathrm{~s}$ in advance.

\section{B. Impact of non updated topology}

In all simulations presented so far, topological changes have been reflected without delay in the Jacobian computation. Additional tests have been performed to assess the impact of 


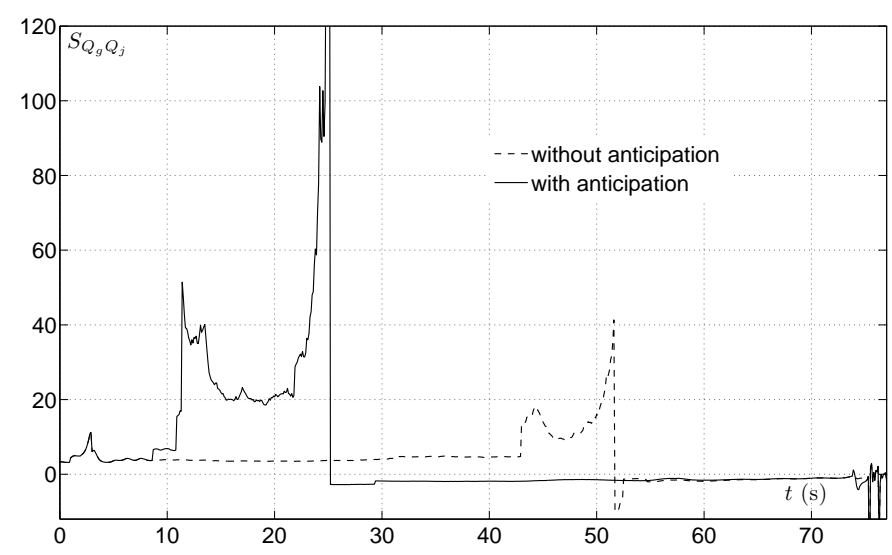

Fig. 14. Sensitivity $S_{Q_{g} Q_{j}}$ at bus 1041 with and without anticipation of OEL activation; Case A with $50 \%$ motor load

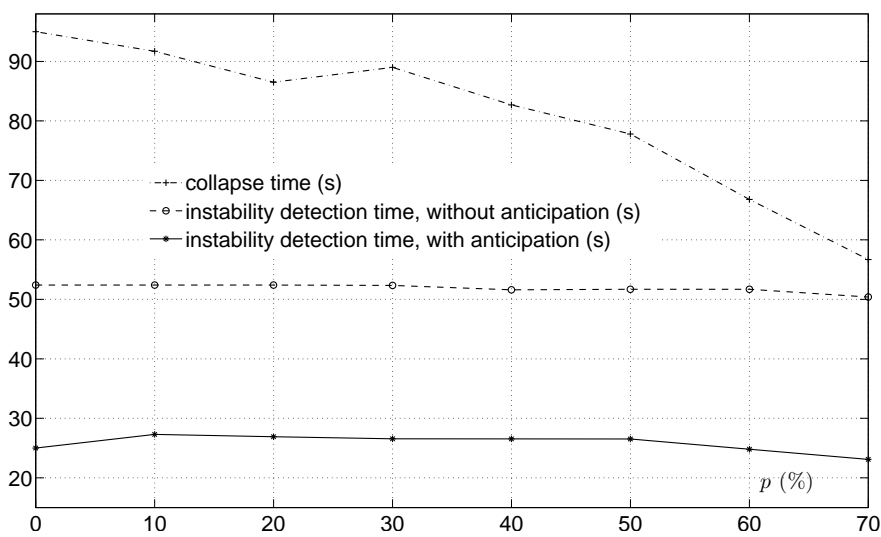

Fig. 15. Collapse and instability detection times, with and without anticipation of OEL activation, for various proportions $p$ of motor load; case A

receiving this information with some delay from the SCADA system. Delays ranging from one to several seconds have been examined, and in view of the good results obtained, no update at all (breaker status change unreported) has been also considered. Figure 16 compares the sensitivity evolutions with the branch outage accounted without delay and not accounted at all. Without topology update, the sensitivities assume smaller values but the difference in terms of detection time is surprisingly negligible. It was found more important to update the model with respect to the (anticipated) OEL status.

The sensitivity to topology errors might be more pronounced in other systems, and it is advisable to update the topology. However, an update at SCADA (instead of PMU) rate seems to be quite acceptable.

\section{Dealing with partial observability of system}

The main requirement of the method is the availability of a PMU configuration making all bus voltages observable in the system.

It is of interest to check the method when observability is limited to the region prone to voltage instability. To this purpose, tests have been performed with a simplified Jacobian computed as follows:

- in the Central and South areas, bus voltages are updated from simulated measurements as previously. This

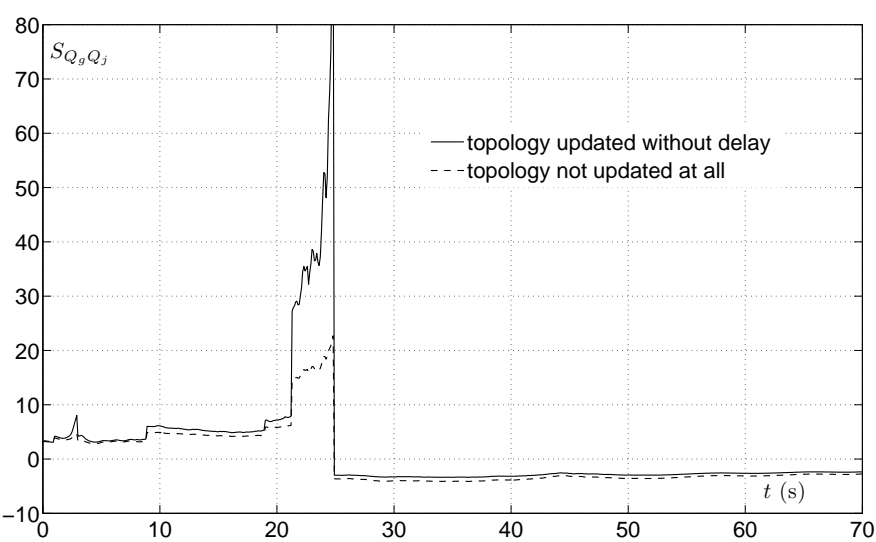

Fig. 16. Impact of non updating topology; case A

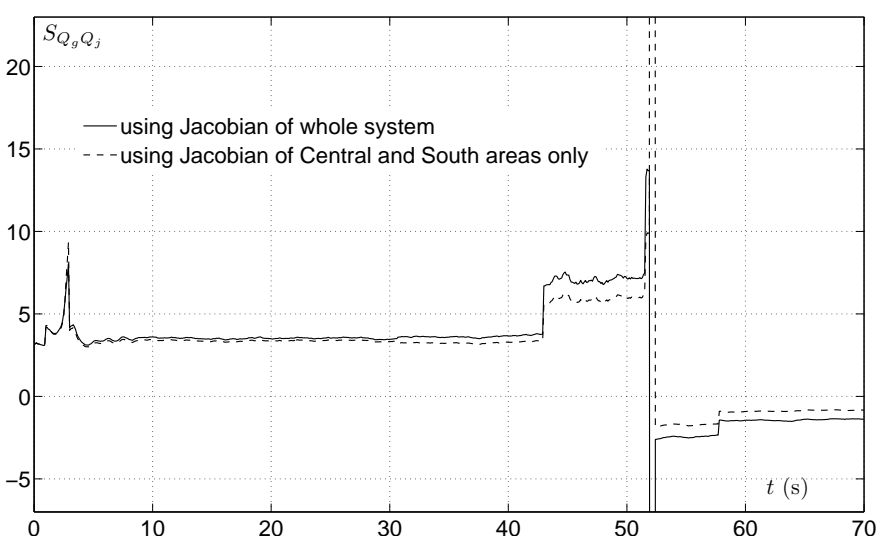

Fig. 17. Evolution of sensitivity at bus 1041, with the Jacobian built for the whole or a part of a system; case A

includes the boundary buses 4021, 4031 and 4032 (see Fig. 1);

- in the remaining of the system, the topology is assumed to be known (from SCADA) but all voltages are set to a fictitious value, obtained (at each time step) as the average of the above three boundary bus voltages. This simplification is inspired of [10].

Even with this drastic simplification, the instability is still correctly identified, as shown in Fig. 17. No significant delay affects the detection time. One can just observe that the sensitivity is lower in magnitude after changing sign.

The unobservable parts would be replaced by more refined equivalents, possibly derived from SCADA measurements. This could prove useful to deal with unobservable parts inside the region of interest.

On the basis of all sensitivity evolutions obtained so far, a proper choice of the detection thresholds in Eq. (14) of the companion paper [1] is $d_{+}=5$ and $d_{-}=-2$, respectively.

\section{COMPARISON With THÉvENIN IMPEDANCE MATCHING CRITERION}

This section provides a comparison of the proposed sensitivities with the Thévenin Impedance Matching (TIM) criterion based on local measurements of voltage and current phasors. 
In the original method proposed in [11] the measurements collected at one load bus are used to obtain the Thévenin equivalent of the system seen from this bus, as well as the apparent impedance of the load. The parameters of the Thévenin equivalent are estimated using a least-square method once two or more sets of measurements are available. The same concept is used in [12], [13] but proximity to instability is expressed in terms of power margin, while a feasibility study of voltage security monitoring and control is reported. A simple method based on two sets of measurements is proposed in [14] to deal with smooth load increases. The recent reference [15] introduces an adaptive algorithm to identify the Thévenin equivalent with higher precision and fast sampling rate, to deal with fast changing operating conditions.

The extension described in [16] involves measurements taken at neighbouring load buses to assist the estimation of the Thévenin equivalent. Similarly, to avoid the time delay of least-square estimation, it is proposed in [17], [18] to obtain the Thévenin equivalent from measurements taken synchronously at both ends of a transmission corridor.

The TIM approach is not free from difficulties. One, of theoretical nature, has to do with the the adequacy of observing a single load when maximum power is reached over a set of loads. It has been shown in [19] that the TIM condition is necessarily met after the maximum load condition that is reflected into Jacobian singularity. Also, the Thévenin impedance has to be estimated from measurements gathered over a time window that should be wide enough for the operating conditions to change, but narrow enough to satisfy the constant Thévenin impedance assumption. This is particular significant when dealing with large disturbances, as in this paper. These and other issues are discussed in [20].

\section{A. Recursive Least Squares}

We concentrate on single-bus Thévenin equivalent. We use the following formulation, taken from [21]. The relation between the measured voltage, the measured current and the Thévenin parameters at time sample $k$ is:

$$
\mathbf{z}_{k}=\mathbf{H}_{k} \mathbf{x}
$$

with:

$$
\mathbf{x}=\left[\begin{array}{c}
E_{x} \\
E_{y} \\
R \\
X
\end{array}\right] \quad \mathbf{z}_{k}=\left[\begin{array}{c}
V_{x k} \\
V_{y k}
\end{array}\right] \quad \mathbf{H}_{k}=\left[\begin{array}{cccc}
1 & 0 & -I_{x k} & I_{y k} \\
0 & 1 & -I_{y k} & -I_{x k}
\end{array}\right]
$$

where $E_{x}$ and $E_{y}$ are the real and imaginary components of the Thévenin e.m.f., and similarly for the current $I$ and bus voltage $V . R$ and $X$ are the Thévenin resistance and reactance, respectively.

Recursive Least Squares (RLS) are used to estimate the time-varying value of $\mathbf{x}$. The estimate at time $k$ is obtained as:

$$
\begin{aligned}
\text { with } & \mathbf{K}_{k}=\mathbf{P}_{k-1} \mathbf{H}_{k}^{T}\left[\lambda \mathbf{I}+\mathbf{H}_{k} \mathbf{P}_{k-1} \mathbf{H}_{k}^{T}\right]^{-1} \\
\text { and } \quad \mathbf{P}_{k} & =\frac{1}{\lambda}\left[\mathbf{I}-\mathbf{K}_{k} \mathbf{H}_{k}\right] \mathbf{P}_{k-1}
\end{aligned}
$$

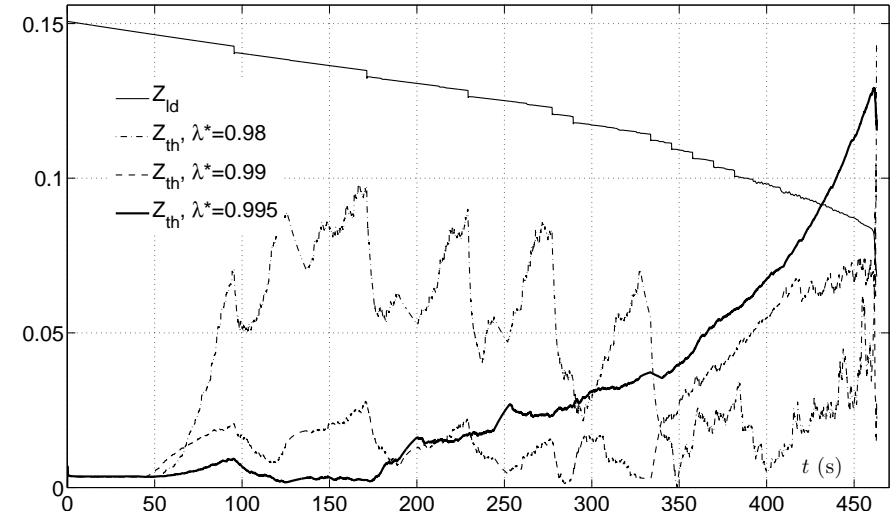

Fig. 18. Evolution of load $\left(Z_{l d}\right)$ and Thévenin $\left(Z_{t h}\right)$ impedances during load increase

where I denotes an identity matrix. $\lambda<1$ is the forgetting factor, aimed at assigning lower weights to older measurements, in order to adjust to changing conditions.

\section{B. Tuning the TIM criterion}

Proper implementation of RLS requires some care to avoid the so-called "wind-up" problem caused by matrix $\mathbf{P}$ when there is too little change in system states [20]. On the other hand, fast changes in the Thévenin parameters have to be tracked by selecting a proper value of $\lambda$.

For a fair comparison, we selected the best possible value of $\lambda$. To this purpose, we considered system conditions for which the TIM criterion has been advocated to work well: a slow load increase scenario [11]. Furthermore, we checked TIM at bus 1041, which is known from sensitivities to be the most critical. Hence, the load at bus 1041 was provisionally converted to constant power and increased at constant rate up to reaching instability.

The RLS scheme is rather sensitive to the choice of the initial values of $\mathbf{x}$, and no-load conditions (at bus 1041) were found to provide the best initial values.

All simulations were performed with the $N(0,0.01)$ noise added to the voltage components. For the sake of comparison, the same noisy voltage values were used in both methods. Since the MA filter on the input voltages proved useful, it was also used in the TIM method.

An adaptive RLS scheme was used, in which $\lambda$ was set to 1 when $\left|V_{k}-V_{k-1}\right|<0.01 \mathrm{pu}$, and to $\lambda^{\star}<1$ otherwise.

Figure 18 shows the time evolution of the load and Thévenin impedances during in the above mentioned load increase scenario, for $\lambda^{\star}=0.95,0.98$ and 0.995 , respectively. It is clearly seen that the smoothest and most consistent evolution of the Thévenin impedance is obtained for $\lambda^{\star}=0.995$. This value was used in the simulations reported hereafter.

\section{Comparison with the proposed sensitivities}

A comparison between sensitivity and TIM methods is of interest even in the above simple load increase scenario. The evolution of various sensitivities for this case is shown in Fig. 19. Instability is clearly detected at $t=285 \mathrm{~s}$. With 


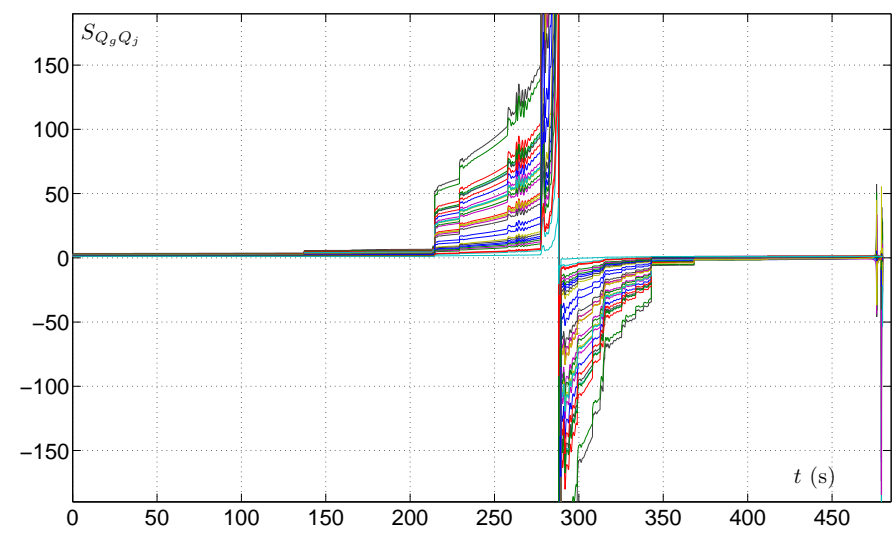

Fig. 19. Evolution of sensitivities during load increase

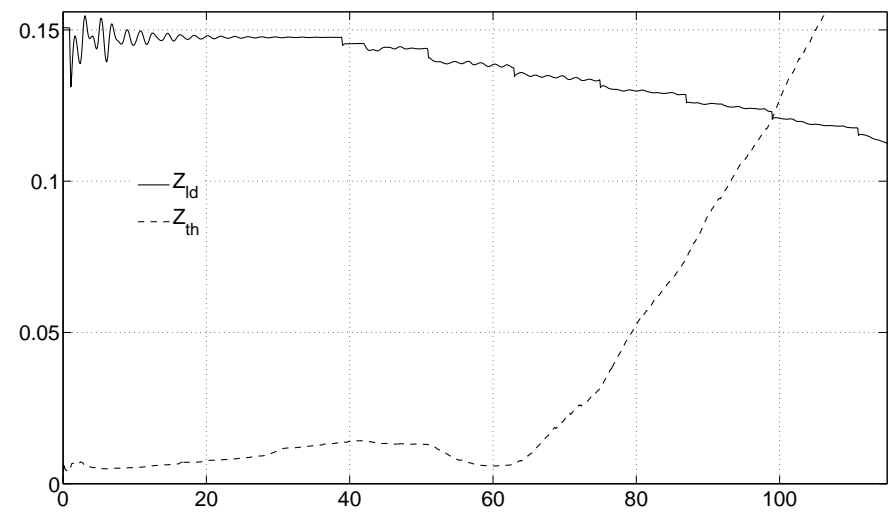

Fig. 20. Evolution of load $\left(Z_{l d}\right)$ and Thévenin $\left(Z_{t h}\right)$ impedances; Case A

the TIM method, instability is declared when the load and impedance curves cross each other. It is seen from Fig. 18 that this occurs at $t=430 \mathrm{~s}$ only, i.e. 145 seconds later. Anticipation is thus much worse.

We now compare the two methods in the large-disturbance scenarios of main interest in this paper. The same sequences of filtered $\mathbf{v}_{x}$ and $\mathbf{v}_{y}$ values were used in both methods, the load at bus 1041 having thus its original behaviour. In cases involving short-circuits, the voltage and current data during fault-on conditions were ignored and replaced by prefault ones when estimating the Thévenin impedance, since we observed that these data cause strong and long-lasting biases in the RLS results that delay instability detection. Let us recall that this is not needed with the proposed sensitivity method, in so far as the delay $\tau$ is applied before declaring instability and even more if the MA filter is used.

Figure 20 shows the evolution of the load and Thévenin impedances in the previously described Case A. These results are to be compared with the ones in Fig. 10. It is easily seen that the TIM approach is inferior in terms of detection time since the sensitivities trigger the alarm some 75 seconds earlier. Furthermore, at the time the load and Thévenin curves cross each other, some transmission voltages are already as low as 0.83 pu (see Fig. 2). Hence, the advantage of the TIM criterion over a mere undervoltage check is not obvious.

Similar results and conclusion are drawn in the marginally unstable Case B, whose results for TIM are shown in Fig. 21,

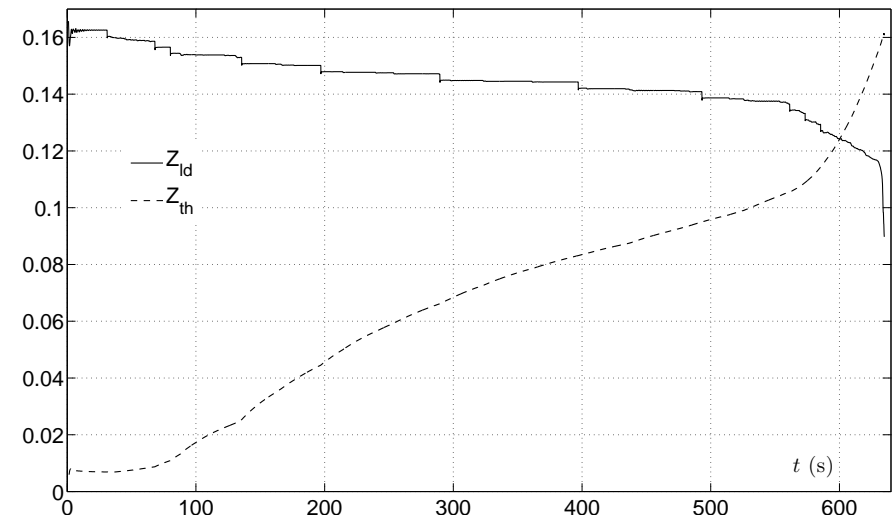

Fig. 21. Evolution of load $\left(Z_{l d}\right)$ and Thévenin $\left(Z_{t h}\right)$ impedances; Case B

to be compared with Fig. 11. Expectedly, the anticipation capability of sensitivities is even larger in this scenario, with an alarm triggered 300 seconds before TIM. Again, the TIMbased alarm is issued when voltages are already low.

\section{CONCLUSION}

The results presented in this paper confirm the ability of the proposed sensitivity computation to early detect an impending voltage instability, well before abnormally low voltages are observed.

The tests were performed on a small system but were deemed demanding. For instance, the simulation of the line outage causing long-term voltage instability included the initiating fault. Also, various levels of measurement noise were considered. Both contribute to making the system deviate from short-term equilibrium (as assumed in the sensitivity computation) but provision is made for these deviations by: (i) processing the input measurements through a moving average, and (ii) waiting for sensitivities to remain negative for some time before issuing an alarm. If these safeguards were not sufficient, one could envisage inhibiting the computations during a short period of time following the detection of large transients (e.g. through a change in circuit breaker position).

Admittedly, the success relies on the availability of a PMU configuration ensuring observability of the region prone to voltage instability. Hopefully, the wide-area monitoring capability offered by such a measurement configuration would serve other objectives of real-time monitoring and control.

While much less demanding in terms of PMU infrastructure, the Thévenin impedance matching criterion has been found to require careful tuning of its parameters, and has shown significantly poorer anticipation capabilities in the large-disturbance scenarios of concern here.

At this point, several directions of future research and testing appear to be of interest:

- in so far as PMU outputs are simulated, more realistic measurement noise could be contemplated, including bias and bad data [7], [22];

- tests are under progress to further assess performance under various motor load assumptions and robustness to network and machine parameter errors; 
- equivalents could be devised to deal with unobservability, especially inside the monitored region (for instance, at lower voltage levels). Such equivalents could be updated at lower rate from SCADA state estimator outputs;

- the advantage brought by the early switching from AVR to OEL control equations has been demonstrated. Even more anticipation could be obtained by forecasting the near-future system states (instead of assessing the current one). This would, however, require a reliable load response model, which is not needed so far;

- the final objective being to devise improved system integrity protection schemes, emergency control must be embedded in the proposed framework. The proposed sensitivities reliably point out the proper location of emergency actions. A closed-loop scheme acting in successive steps and observing the effect of its actions (as shown in [23] in the case of undervoltage load shedding) appears to be attractive.

\section{REFERENCES}

[1] M. Glavic and T. Van Cutsem, "Wide area detection of voltage instability from system states provided by PMUs. Part I: Principle," companion paper submitted for publication in IEEE Trans. Power Syst.

[2] M. Stubbe (Convener), Long-Term Dynamics - Phase II, Report of CIGRE Task Force 38.02.08, Jan. 1995.

[3] T. Van Cutsem and C. Vournas, Voltage Stability of Electric Power Systems, Boston, Springer (previously Kluwer Academic Publishers), 1998.

[4] C. D. Vournas, E. G. Potamianakis, C. Moors, and T. Van Cutsem, "An educational simulation tool for power system control and stability," IEEE Trans. on Power Syst., vol. 19, No 1, pp. 48-55, Feb. 2004.

[5] F. Capitanescu and T. Van Cutsem, "Unified sensitivity analysis of unstable or low voltages caused by load increases or contingencies," IEEE Trans. on Power Syst., vol. 20, no. 1, pp. 321-329, Feb. 2005.

[6] A. P. Sakis Meliopoulos, G. J. Cokkinides, F. Galvan, and B. Fardanesh, "Distributed State Estimator - Advances and Demonstration," in Proc. 2008 41th International Conference on System Sciences, Hawaii, Jan. 2008.

[7] M. M. Begovic, D. Novosel, and B. Djokic, "Issues Related to the Implementation of Synchrophasor Measurements," in Proc. 2008 41th International Conference on System Sciences, Hawaii, Jan. 2008.

[8] B. Genet and J-C. Maun, "Voltage Stability Monitoring using WideArea Measurement System," in Proc. IEEE Power Tech Conf., Lausanne, Switzerland, paper 392, Jun. 2007.

[9] C. W. Taylor, Power System Voltage Stability, EPRI Power System Engineering Series, McGraw Hill, 1994.

[10] M. M. Begovic and A. G. Phadke, "Voltage Stability Assessment through Measurement of a Reduced State Vector," IEEE Trans. on Power Syst., vol. 5, no. 1, pp. 198-203, Feb. 1990.

[11] K. Vu, M. M. Begovic, D. Novosel, and M. M. Saha, "Use of Local Measurements to Estimate Voltage Stability Margin," IEEE Trans. on Power Syst., vol. 14, no. 3, pp. 1029-1035, Aug. 1999.

[12] K. Vu, M. D. Julian, J. O. Gjerde, N. Bhatt, B. Laois, and R. Schulz, "Voltage Instability Predictor (VIP) and its Applications," in Proc. 1999 13th Power System Computation Conference (PSCC), Trondheim, Norway, Jun.-Jul. 1999, pp. 308-313.
[13] D. E. Julian, R. P. Schulz, K. T. Vu, W. H. Quaintance, N. B. Bhatt, and D. Novosel, "Quantifying proximity to voltage collapse using the voltage instability predictor (VIP)," in Proc. 2000 IEEE Power Eng. Soc. Summer Meeting, Seattle, WA, Jul. 2000, pp. 16-20.

[14] I. Smon, G. Verbic, and F. Gubina, "Local Voltage-Stability Index Using Tellegen's Theorem," IEEE Trans. on Power Syst., vol. 21, no. 3, pp. 1267-1275, Aug. 2006.

[15] S. Corsi and G. N. Taranto, "A Real-Time Voltage Instability Identification Algorithm Based on Local Phasor Measurements," IEEE Trans. on Power Syst., vol. 23, no. 3, pp. 1271-1279, Aug. 2008.

[16] L. Warland and A. T. Holen, "Estimation of Distance to Voltage Collapse: Testing and Algorithm Based on Local Measurements," in Proc. 2002 14th Power System Computation Conference (PSCC), Paper s38p03, Sevilla, Spain, Jun. 2002.

[17] C. Rehtanz and J. Bertsch, "Wide Area Measurement and Protection System for Emergency Voltage Stability Control," in Proc. 2002 IEEE Power Eng. Soc. Winter Meeting, New York, NY, 2002, pp. 842-847.

[18] Zima M., Larsson M., Korba P., Rehtanz C., and Andersson G., "Design aspects for wide-area monitoring and control systems," Proceedings of the IEEE, vol. 93, no. 5, pp. 980-996, May 2005.

[19] C. D. Vournas and N. G. Sakellaridis, "Tracking Maximum Loadability Conditions in Power Systems," in Proc. 2007 Bulk Power System Dynamics and Control-VII, Paper 104, Charleston, SC, Aug. 2007.

[20] B. Genet, T. Sezi, and J-C. Maun, "Comparison of Thevenin's Equivalent Based Methods to Monitor Voltage Stability," in Proc. 2008 16th Power System Computation Conference (PSCC), Glasgow, UK, paper 460, Jul. 2008.

[21] B. Milosevic and M. Begovic, "Voltage Stability Protection and Control using a Wide-Area Network of Phasor Measurements," IEEE Trans. on Power Syst., vol. 18, no. 1, pp. 121-127, Feb. 2003.

[22] M. M. Adibi, R. J. Kafka, K. A. Clements, and J. P Stovall, "Integration of remote measurement calibration with state estimation - A feasibility study," IEEE Trans. on Power Syst., vol. 7, no. 3, pp. 1164-1172, Aug. 1992.

[23] B. Otomega and T. Van Cutsem, "Undervoltage load shedding using distributed controllers," IEEE Trans. on Power Syst., vol. 22, no. 4, pp. 1898-1907, Nov. 2007.

Mevludin Glavic (M'04-SM'07) received the M.Sc. and Ph.D. degrees from the Univ. of Belgrade (Serbia) and Tuzla (Bosnia) in 1991 and 1997, respectively. He spent the academic year 1999-2000 with the Univ. of WisconsinMadison, as a Fulbright postdoctoral scholar. From 2001 to 2004, he has been a Senior Research Fellow at the Univ. of Liège. Since then he has been working as consultant for Deling-doo (Bosnia) in projects with AREVA T\&D (France) and Suez Tractebel Eng. (Belgium), as well as adjunct professor at the Dept. of Mathematics of the Univ. of Tuzla. He is presently a visiting professor at the Univ. of Liège, with a grant from the Fund for Scientific Research (FNRS). His research interests are in power system dynamics, stability, security, optimization, and real-time control.

Thierry Van Cutsem (F'05) graduated in Electrical-Mechanical Engineering from the University of Liège (Belgium), where he obtained the Ph.D. degree and he is now adjunct professor. Since 1980, he has been with the Fund for Scientific Research (FNRS), of which he is now a Research Director. His research interests are in power system dynamics, stability, security, simulation and optimization, in particular voltage stability and security. 